\title{
EDITORIAL AND COMMENT \\ Gender Differences in Experiences of K-Awardees: Beyond Space, Resources and Science
}

\author{
Amparo C. Villablanca, $M D^{7}$ and Lydia P. Howell, MD² \\ 'Department of Internal Medicine, Division of Cardiovascular Medicine, University of California, Davis, CA, USA; ${ }^{2}$ Department of Pathology, \\ University of California, Davis, CA, USA.
}

J Gen Intern Med 30(4):381-3

DOI: $10.1007 / \mathrm{s} 11606-014-3177-2$

(c) Society of General Internal Medicine 2015

$\mathrm{T}$ he thought-provoking article by Dr. Holliday and colleagues highlights gender differences among recipients of K awards. Even though these awards include provisions for protected time and resources, a higher proportion of women awardees reported inadequate access to "soft" resources, notably grant administrators and statistical support. The authors suggest that the root cause of this discrepancy may be that women are disadvantaged in the informal negotiations necessary to achieve these less structured resources, either due to an inadequate or less effective skill set, or due to unconscious bias by those with whom they negotiate. The authors further imply that inadequacy of research support may underlie gender discrepancies in publication productivity and securing research funding, and that academic productivity may be inhibited by unfair treatment. Strengths of the study include the robust survey response rate $(75 \%)$, the number of awardees studied $(1,708)$, and the statistical methodology that controlled for confounders, including academic rank.

Holliday and colleagues' findings reinforce many of the conclusions from the National Academies' landmark report, Beyond Bias and Barriers, ${ }^{1}$ which drew attention to similar factors that can adversely impact women's careers in biomedical sciences. Beyond Bias and Barriers specifically noted access to resources, as well as personal and professional development (e.g., negotiation skills), as contributing factors to the attrition of women from careers in science and academia. Other factors included in the report were mentorship/ sponsorship, and the challenges of balancing career and family responsibilities. Though Beyond Bias and Barriers was published nine years ago, the Holliday article makes it clear that these issues still remain significant today. Holliday et al. also draws to our attention the effect of the implicit (unconscious) biases that are common in the workplace, as well as sex stereotype threat. As articles by Carnes et al. ${ }^{2}$ and Easterly and Ricard ${ }^{3}$ point out, these biases lead to micro-inequities that, especially for women, can erode academic success over time and discourage women in academic medical careers, and

Published online February 10, 2015 that both are important contributors to the attrition of women from academics.

Data from the Association of American Medical Colleges (AAMC)'s recently released report entitled The State of Women in Academic Medicine (https://www.aamc.org/data/ databook/tables/accessed November 10, 2014) demonstrate that the pipeline has long been flush with women who have the appropriate terminal degrees to enter careers in academic medicine. Yet their data also show that women currently make up a little more than one-third (38\%) of full-time academic medicine faculty. Under-representation persists for full-time women assistant, associate and full professors (44, 34 and $21 \%$, respectively), and the percentage of women in academic medicine in top leadership positions, such as department chairs and deans, remains low (15 and $16 \%$, respectively). The same report highlights that despite modest progress in the proportion of new faculty hires who are women (up $4 \%$ since 2008), the steady attrition of women out of the academic advancement pipeline has remained essentially unchanged for over 25 years, ${ }^{4}$ and that the proportion of faculty departures who are women is rising (up $5 \%$ since 2008). Successful career progression requires interaction of positive influences on both the individual and institutional levels, since each can influence a faculty member's available time, resources, drive, and productivity. Many obstacles are encountered by men and women faculty during their academic careers, determined by individual, family, and institutional/societal influences. Although some of the same problems affect both sexes (as summarized in the article by Holliday), it is important to focuses on the special issues facing women as they contribute to differential outcomes. ${ }^{5,6}$ Holliday et al.'s article is an important contribution, since it highlights issues that should be fairly easy to address.

Although demand for family care responsibilities and career satisfaction were not assessed in the study by Holliday et al., other studies have shown that women with children have less institutional support, fewer publications, slower self-perceived career progress, and lower career satisfaction. ${ }^{5,7}$ Given that in the study of K awardees, the majority of K-23 awards (clinician scientist) were held by women (64\%) and the majority of K-08 awards (basic scientist) were held by men (60\%), it would be of interest to know the extent to which differences in the types of awards and their demands played a role in the findings of the study. 
Women are often the canaries in the coal mine. Though women were impacted more in Holliday et al.'s study, we find it interesting that men also reported feeling disadvantaged regarding resources. Men and women equally made requests for support that were often only partially fulfilled, and both genders reported experiencing unfair treatment. Our own work ${ }^{8}$ has identified vulnerable faculty groups for career dissatisfaction, one of which is male faculty in the early career stage. The inherent conflicts and stressors affecting satisfaction of early career faculty may be similar for both genders, due to changing generational values for family, worklike integration, and career flexibility. ${ }^{9}$ Other studies have also shown that a substantial proportion of both men and women report that conflicts between work, family and career are significant determinants of career dissatisfaction and a strong predictor of leaving a career in academic medicine. ${ }^{10}$ Recent work by the Work Family Institute demonstrates that there is growing work-life conflict among men in the younger generations and that men are stigmatized when they seek solutions typically utilized by women, such as flexible work schedules.

If left unaddressed, the unmet needs of early career faculty are potentially significant threats to a successful academic medical workforce. A number of approaches have been proposed and are being utilized to support the careers of women faculty. ${ }^{11}$ They include attending to generational issues and career flexibility in academia; building multigenerational team environments; developing policies aimed at improving work-life compatibility to lessen conflict; creating a supportive culture; providing adequate compensation and rewards for productivity; aligning demands placed on faculty with strategic priorities; and supporting research careers. The ability of academic medical centers to retain women faculty is particularly important to excellence in patient care, teaching and research, to ensure appropriate role modeling for junior faculty, and to provide for diversity at all levels, including leadership. Action steps to advance women faculty at academic medical centers include advocacy and support for women faculty and women leaders, and mentoring and coaching programs that include men in the conversation about how to mentor and advance women. It is as yet unclear whether the approaches currently being implemented at academic health systems nationwide will help to stem the tide of women exiting academic careers. The findings of the work of Holliday et al. suggest several directions for future research and organizational change: The evidence base is needed to determine whether deficiencies in research support result from difficulties some women have with informal negotiations, and whether providing directed skill building in communication and negotiation can impact career outcomes; similarly, if unconscious biases predominate in less structured settings as suggested by the article by Holliday et al., effective attitudinal change models should be developed and tested, utilizing education, role play, feedback or other techniques with promise for delivering change, such as those in the AAMC's learning lab (https://www.aamc. org/in itiatives/diversity/322996/ lablearningonunconsciousbias.html, accessed November 10, 2014).

Data and policies alone may not be enough to address the unfair treatment reported by women, and to a lesser extent men, in the work of Holliday and colleagues. Institutional leadership, sustained efforts, and actionable strategies will be necessary at all levels to reform workplace climate and culture, and to address systematic constraints, in order to recognize and address the elements of institutional life that may disproportionately disadvantage women. Additional approaches yet to be tested include institutional practices that promote equity, 'lean in' circles and networks for professional support, and the outcomes of current best practices. In addition, it is as yet unclear whether current trends favoring team-based science and resource sharing will favorably impact women's careers and their advancement, and data-driven efforts are needed to demonstrate results in this realm. While we recognize the need for additional focused research efforts to understand the under-representation of women in academia and the elements of dissatisfaction that lead to departure, it is clear that the pace of progress will need to increase. Now more than ever, the strategic advantage of a satisfied workforce will allow academic institutions to not only train but also attract, retain and advance the top talent that will be critical to meet the workforce, healthcare, scientific, and resource demands of the future.

Conflict of Interest: The authors declare that they do not have a conflict of interest.

Corresponding Author: Amparo C. Villablanca, MD; Department of Internal Medicine, Division of Cardiovascular MedicineUniversity of California, One Shields Ave., TB 172, Davis, CA 95616-8636, USA (e-mail: avillablanca@ucdavis.edu).

\section{REFERENCES}

1. National Academy of Sciences, National Academy of Engineering, Institute of Medicine. Beyond bias and barrier: fulfilling the potential of women in academic science and engineering. Washington DC: The National Academy Press; 2006.

2. Carnes MD, Morrissey C, Geller S. Women's Health and Women's Leadership in Academic Medicine: Hitting the Same Glass Ceiling? J Women's Health. 2008;17(9): 1453-62.

3. Easterly DM, Ricard CS. Conscious efforts to end unconscious bias: why women leave academic research. J Res Admin. 2011;42(1):61-73.

4. Berryman SE. Who will do science: minority and female attainment of science and mathematics degrees: trends and causes. Trends and Causes. New York: Rockefeller Foundation; 1983.

5. Long JE. From scarcity to visibility: gender differences in the careers of doctoral scientists and engineers. National Academy Press; 2001. 
6. Cropsey KL, Masho Sw, Shiang R, Sikka V, Kornstein SG, Hampton CL. Why do faculty leave? Reasons for attrition of women and minority faculty from a medical school: four year results. J Womens Health. 2008; 17:1111-8.

7. Carr PL, Ash AS, Friedman RH, Caramucci A, Barnett RC, Szalacha L Palepu A, Moskowitz MA. Relation of family responsibilities and gender to the productivity and career satisfaction of medical faculty. Ann Intern Med 1998;579-580.

8. Howell LP, Beckett L, Nettiksimmons J, Villablanca AC. Generational and Gender Perspectives Toward Career Flexibility: An
Approach to Ensuring the Faculty Workforce of the Future. Am J Med (APM Perspect). 2012;25(7):719-27.

9. Bickel J, Brown AJ, Generation X. Implications for faculty recruitment and development in academic health centers. Acad Med. 2005;80:203-4.

10. Nonemaker L. Women physicians in academic medicine: new insights from cohort studies. NEJM. 2000;399-405.

11. Villablanca AC, Beckett L, Nettiksimmons J, Howell LP. Career flexibility and family-friendly policies: an $\mathrm{NIH}$-funded study to enhance women's careers in biomedical sciences. J Womens Health. 2011;20:148596. 